\title{
Transjugular intrahepatic portosystemic shunt (TIPS) versus endoscopic variceal ligation in the prevention of variceal rebleeding in patients with cirrhosis: a randomised trial
}

G Pomier-Layrargues, J-P Villeneuve, M Deschênes, B Bui, P Perreault, D Fenyves, B Willems, D Marleau, M Bilodeau, M Lafortune, M-P Dufresne

\begin{abstract}
Background and aims-The transjugular intrahepatic portosystemic shunt (TIPS) is a new therapeutic modality for variceal bleeding. In this study we compared the two year survival and rebleeding rates in cirrhotic patients treated by either variceal band ligation or TIPS for variceal bleeding.

Methods-Eighty cirrhotic patients (Pugh score 7-12) with variceal bleeding were randomly allocated to TIPS $(n=41)$ or ligation $(n=39), 24$ hours after control of bleeding.

Results-Mean follow up was 581 days in the ligation group and 678 days in the TIPS group. The two year survival rate was $57 \%$ in the TIPS group and $56 \%$ in the ligation group (NS); the incidence of variceal rebleeding after two years was $18 \%$ in the TIPS group and $66 \%$ in the ligation group $(p<0.001)$. Uncontrolled rebleeding occurred in 11 patients in the ligation group (eight were rescued by emergency TIPS) but in none of the TIPS group. The incidence of encephalopathy at two years was $47 \%$ in the TIPS group and $44 \%$ in the ligation group (NS).

Conclusions-TIPS did not increase the two year survival rate compared with variceal band ligation after variceal bleeding in cirrhotic patients with moderate or severe liver failure. It significantly reduced the incidence of variceal rebleeding without increasing the rate of encephalopathy.

(Gut 2001;48:390-396)
\end{abstract}

Keywords: portal hypertension; cirrhosis; variceal bleeding; transjugular intrahepatic portosystemic shunt; variceal band ligation

Division,

Royal-Victoria

Hospital and McGill

University, Montréal,

Canada

$M$ Deschênes

Correspondence to:

Dr G Pomier-Layrargues,

Centre de recherche du

CHUM, Hôpital Saint-Luc,

264, East René-Lévesque

blvd, Montréal, Québec,

Canada H2X 1P1.

hepato.saint-luc@sympatico.ca

Accepted for publication

5 September 2000

Variceal bleeding is a leading cause of death in cirrhotic patients. Mortality following an episode of variceal haemorrhage without treatment is approximately $50 \%$ at three months and $66 \%$ at one year. ${ }^{1}$ This high mortality rate can be explained mainly by two factors. Rebleeding from varices is a major cause of death in these patients; the incidence of rebleeding is $35 \%$ at six weeks after the initial bleeding episode and $75 \%$ at one year. ${ }^{2}$ The occurrence of liver failure, usually precipitated by the bleeding episode, is also a major cause of death. In this respect the quality of liver function, as evaluated by the Pugh score, ${ }^{3}$ has long been recognised as a major determinant of survival, with patients with good liver function (Pugh class A) having a much better prognosis than those with moderate or severe liver failure (Pugh classes B and C). ${ }^{4}$

It should also be noted that the survival slope following variceal rupture is not linear: the risk of rebleeding and the risk of dying are much higher during the initial three months after the index bleeding episode. This is followed by a long phase in which the risk of dying decreases and is primarily dependent on the severity of the underlying liver disease. Smith and Graham $^{1}$ demonstrated that this early phase mortality is the prime determinant of the long term survival slope. Thus any significant improvement in long term survival must rely on manoeuvres which will improve survival in the early period.

Various treatments have been proposed to prevent rebleeding for ruptured varices in the hope of improving survival. Emergency portacaval shunt performed within 48 hours after the index bleed could be a good treatment for patients with variceal bleeding. ${ }^{5}$ However, this operation is associated with an unacceptably high postoperative mortality in decompensated cirrhotic patients. ${ }^{6}$ Vasoactive drugs used alone or in combinations (namely propranolol and isosorbide monotritrate) can prevent rebleeding in a significant number of cirrhotic patients, and a recent meta-analysis has shown that propranolol improves survival. ${ }^{7-9}$

Endoscopic sclerotherapy decreases the rate of variceal rebleeding and reduces the number of deaths by $25 \% .{ }^{10}$ More recently, endoscopic ligation of varices has been shown to be superior to sclerotherapy in terms of rebleeding rate, complications, and number of sessions required to achieve variceal obliteration. ${ }^{11}$

Transjugular intrahepatic portosystemic
(IIPS) can be used as an emergency procedure in patients with bleeding varices; it can be performed in Child-Pugh class C patients in whom shunt surgery is usually considered to be contraindicated. ${ }^{12}$

The purpose of this single centre randomised controlled trial was to compare the efficacy of TIPS with that of endoscopic variceal ligation on survival following an

Abbreviations used in this paper: TIPS, transjugular intrahepatic portosystemic shunt. 
episode of variceal bleeding in cirrhotic patients with moderate or severe liver disease.

\section{Patients and methods}

PATIENT SELECTION

Between February 1994 and September 1997, 158 patients with cirrhosis (according to clinical findings, laboratory data, ultrasonography, and/or liver biopsy), aged 18-75 years, with an episode of variceal haemorrhage demonstrated by upper gastrointestinal endoscopy performed within 72 hours of the bleeding episode and with a Pugh score of 7-12 were considered for inclusion in the study. Eighty patients were included in the study and 78 patients with any of the following criteria were excluded: portal vein thrombosis as shown by echo-Doppler and/or arteriography $(n=7)$; previous treatment with endoscopic sclerotherapy or ligation within three months $(n=24)$; previous shunt surgery $(n=1)$; bleeding from large fundal varices $(n=8)$; presence of hepatocellular carcinoma $(n=13)$; cardiac, respiratory, or renal failure $(n=5)$; non-compliance $(n=6)$; sepsis $(n=3)$; uncontrolled haemorrhage $(n=4)$; three patients were excluded because they were on a waiting list for emergency liver transplantation, one for concomitant cerebral haemorrhage, one due to anticoagulation, and one for concomitant extrahepatic biliary obstruction; and one patient refused to participate in the study.

\section{RANDOMISATION}

The study protocol was approved by our local ethics committee and informed consent was obtained prior to randomisation from all patients or next of kin.

Initial control of the bleeding episode was obtained by one session of endoscopic ligation or sclerotherapy and/or balloon tamponade and/or octreotide infusion in both groups. Randomisation was carried out only after the initial haemorrhagic episode had been controlled and haemodynamic status had been stable for at least 24 hours. This status was defined as follows: stable pulse, systolic arterial blood pressure greater than $90 \mathrm{~mm} \mathrm{Hg}$, stable haematocrit level, and absence of red blood in the gastric lavage aspirate. Patients were allocated to endoscopic ligation or intrahepatic shunting by balanced randomisation using the sealed envelope method. ${ }^{13}$

\section{TREATMENT}

Endoscopic variceal ligation

In patients assigned to the ligation group, ligation was performed on days 1 and 10 and then every 3-4 weeks until obliteration of varices occurred. All patients then underwent follow up endoscopy every three months. Recurrence of varices was treated by further ligations. All procedures were performed by one of five experienced endoscopists (GPL, BW, MB, DF, or JPV).

\section{Intrahepatic portosystemic stent shunting}

Patients assigned to the shunting group underwent the procedure within 72 hours of randomisation. TIPS was performed by one of three authors skilled in this procedure (GPL, $\mathrm{MD}$, or $\mathrm{BB}$ ).

A 24 hour antibioprophylaxis was provided but no anticoagulation was used. The portal vein was catheterised using the transjugular approach, as previously described. ${ }^{14}$ After catheterisation, the tract between the portal vein and hepatic vein was dilated up to $10 \mathrm{~mm}$ using balloon angioplasty catheters; thereafter a stent was inserted to create the shunt. Insertion of two or more stents was needed in selected cases because of the length of the tract between the hepatic vein and portal vein. Shunting was aimed at decreasing the portocaval gradient below $12 \mathrm{~mm} \mathrm{Hg}$ to avoid rebleeding. The patency of the shunt was verified using duplex Doppler ultrasonography at 24 hours and then every three months over two years. The portocaval gradient (portal pressure-inferior vena cava pressure) was measured after cannulation of the portal vein through the stent at two months, one year, and two years.

If Doppler studies suggested shunt dysfunction, as defined by a $20 \%$ decrease in stent flow and/or reversal of the direction of flow in the intrahepatic portal veins or left gastric vein, ${ }^{15}$ stent catheterisation was performed and shunt revision (stent dilatation with or without addition of a new stent) was performed if the portocaval gradient was greater than $12 \mathrm{~mm}$ $\mathrm{Hg}$.

FOLLOW UP

Every subject included in the study was followed until death, loss to follow up, or completion of the study (closure date 15 January 1999). Following discharge from hospital, patients from both groups were followed at regular intervals in a specialised clinic under the coordination of a research nurse. In the TIPS group, patients were evaluated monthly after discharge for three months and then every three months; in the banding group, patients were evaluated every 3-4 weeks at the time of endoscopic treatment and every three months after variceal obliteration (usually after 4-5 sessions of banding). At each visit, patients underwent a brief medical history including a record of possible gastrointestinal bleed, alcohol intake, concurrent medications and patient complaints, a brief physical examination including estimation of ascites and encephalopathy by routine neurological examination, and laboratory profile.

Autopsy was performed where possible in patients who died and the patency of the stent shunt was verified.

\section{DEFINITIONS}

\section{Rebleeding}

Rebleeding was defined as the presence of haematemesis and/or melena with a $20 \mathrm{~g} / 1$ decrease in haemoglobin levels or clinical evidence of hypovolaemic shock. The source of rebleeding was determined by emergency endoscopy.

Variceal rebleeding was treated by shunt revision (with stent dilatation and/or addition of new stents) in the TIPS group and by banding in the ligation group. Failure of treatment 
Table 1 Patients characteristics at randomisation

\begin{tabular}{lll}
\hline & Ligation $(n=39)$ & TIPS $(n=41)$ \\
\hline Age $(\mathrm{y})$ & $54.3(10.9)$ & $52.9(13.3)$ \\
Sex $(\mathrm{M} / \mathrm{F})$ & $27 / 12$ & $29 / 12$ \\
Aetiology of liver disease (n) & & \\
$\quad$ Alcoholism & 24 & 25 \\
Hepatitis B virus & 2 & 3 \\
Hepatitis C virus & 3 & 1 \\
PBC & 3 & 1 \\
Sclerosing cholangitis & 1 & 3 \\
Autoimmune hepatitis & 1 & 0 \\
Haemochromatosis & 1 & 5 \\
Cryptogenic & 4 & 3 \\
Previous variceal bleed (n) & 8 & 6 \\
Previous sclerotherapy (n) & 4 & 8 \\
Associated fundal varices (n) & 6 & $3.4(2.9)$ \\
Blood units (index bleed) & $3.1(2.5)$ & $44(35)$ \\
Time from index bleed to randomisation (hours) & $42(33)$ & $13(11)$ \\
Time from randomisation to TIPS (hours) & & 9 \\
Balloon tamponade & 5 & $9.6(1.6)$ \\
Pugh score & $9.8(1.6)$ & $49(37)^{\star}$ \\
Serum bilirubin ( $\mu$ mol/l) & $89(93)$ & $24.9(4.5)$ \\
Serum albumin (g/l) & $25.0(5.3)$ & $89(17)$ \\
Haemoglobin (g/l) & $91(17)$ & $1.38(0.25)$ \\
Prothrombin (INR) & $1.41(0.28)$ & \\
\hline
\end{tabular}

PBC, primary biliary cirrhosis; INR, international normalised ratio. ${ }^{\star} \mathrm{p}<0.05$.

was defined as the occurrence within two years of three rebleeding episodes or the presence of variceal bleeding which became uncontrollable (need for transfusions despite tamponade with a Blakemore tube). If treatment failure occurred, patients were crossed over to the alternate study group.

Hepatic encephalopathy

Encephalopathy was classified as mild (isolated episodes with a well identified precipitating factor), moderate (recurrent episodes easily managed by diet and/or medication), or severe (not controlled by medical therapy and requiring hospitalisation).

END POINTS

The primary end point of the study was the two year survival rate. Secondary end points were incidence of rebleeding at two years, incidence of treatment failure, and incidence of hepatic encephalopathy during follow up. Sample size was calculated assuming a two year survival rate of $50 \%$ in the ligation group and $80 \%$ in the TIPS group with an estimated dropout rate of $5 \%$. Based on these estimates, inclusion of 39 patients was required to detect a difference with a $\mathrm{p}$ value of $<0.05$ and with $\alpha$ and $\beta$ values of 0.05 and 0.2 , respectively. ${ }^{16}$

\section{STATISTICS}

Data were analysed according to an intention to treat strategy. Patients in whom the treatment could not be performed for technical reasons were assigned for analysis to the treatment group to which they were randomised. Patients who underwent liver transplantation were censored at the date of transplantation. Survival rate, percentage of subjects free of rebleeding, hepatic encephalopathy, and shunt dysfunction during the study period were calculated according to the Kaplan-Meier method for each group, and groups were compared using the log rank test. A multiple logistic regression analysis was performed to determine the magnitude and significance of treatment effect on survival rate and variceal
Table 2 Causes of death in the ligation and transjugular intrahepatic portosystemic shunt (TIPS) groups

\begin{tabular}{lcc}
\hline & $\begin{array}{l}\text { Ligation } \\
(n=16)\end{array}$ & $\begin{array}{l}\text { TIPS } \\
(n=17)\end{array}$ \\
\hline Variceal bleeding & 3 & - \\
Liver failure & 9 & 10 \\
Sepsis & 2 & 2 \\
Hepatocellular carcinoma & - & 3 \\
Haemoperitoneum & - & 1 \\
Heart failure & 1 & 1 \\
Non-hepatic cancer & 1 & - \\
\hline
\end{tabular}

rebleeding rate, and incidence of post-TIPS hepatic encephalopathy while adjusting for other prognostic variables. Differences between the ligation and shunted groups were tested using the $\chi^{2}$ exact test or the Wilcoxon rank order test for non-parametric variables and by analysis of variance for continuous data. Results of the statistical tests were considered to be significant at $\mathrm{p}<0.05$.

\section{Results}

CLINICAL AND LABORATORY DATA

Thirty nine patients were assigned to variceal ligation and 41 to TIPS. All patients underwent endoscopic treatment (ligation or sclerotherapy) at the initial endoscopy on the day of the index bleed. The severity of the index bleed, as reflected by transfusion requirements and need for balloon tamponade, was similar in both groups (table 1). Clinical and biochemical parameters were not significantly different in the two groups with the exception of serum bilirubin level which was significantly higher in the ligation group.

The time interval between the index bleed and randomisation was 42 and 44 hours for the ligation and TIPS groups, respectively. The time interval between randomisation and the TIPS procedure was 13 (11) hours. In the ligation group a second treatment was performed 8-10 days after the initial endoscopy. The number of patients with a history of previous variceal bleeding or non-bleeding fundal varices was similar in both groups.

\section{PROCEDURES}

TIPS was performed successfully in 40 of 41 patients randomised to TIPS. TIPS failure was due to perforation of the portal vein and the patient died before completion of the procedure. A post-TIPS portacaval gradient lower than $12 \mathrm{~mm} \mathrm{Hg}$ was obtained in all but six patients.

Variceal ligation was performed successfully in all patients. Varices were successfully eradicated in 21 patients (10 patients died before eradication, eight patients had TIPS because of endoscopic therapy failure, and one patient underwent orthotopic liver transplantation). The median number of sessions for eradication of varices was four.

SURVIVAL

Seventeen patients died in the TIPS group and 16 in the ligation group. Causes of death are listed in table 2. Two patients in the TIPS group underwent liver transplantation compared with four in the ligation group. 


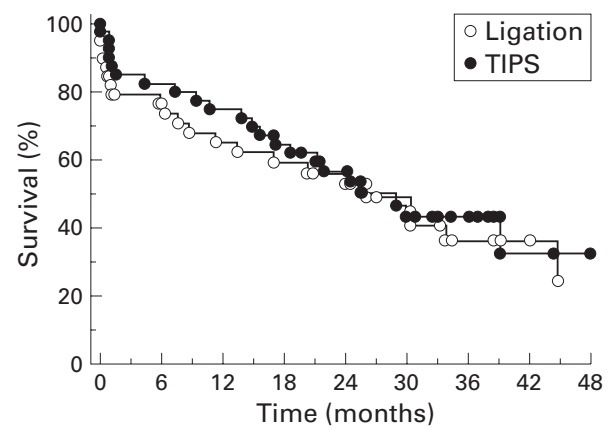

No of patients at risk

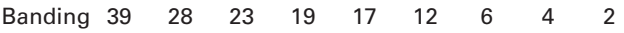

$\begin{array}{llllllllll}\text { TIPS } & 41 & 33 & 30 & 25 & 20 & 13 & 9 & 3 & 2\end{array}$

Figure 1 Cumulative proportion of patients surviving (Kaplan-Meier plot) in the ligation and transjugular intrahepatic portosystemic shunt (TIPS) groups.

Table 3 Causes of gastrointestinal bleeding within two years of randomisation in the ligation and transjugular intrahepatic portosystemic shunt (TIPS) groups

\begin{tabular}{lcc}
\hline Source of bleeding & Ligation & TIPS \\
\hline Varices & 30 & 9 \\
Oesophageal ulcer due to ligation & 7 & 3 \\
Mallory-Weiss & 2 & - \\
Oesophagitis & 2 & 3 \\
Haemobilia & - & 1 \\
Gastropathy & - & 1 \\
Peptic ulcer & 41 & 2 \\
Total number of episodes & & 18 \\
\hline
\end{tabular}

The cumulative two year survival rate was not significantly different between the TIPS and ligation groups $(56.8 \%$ v $52.9 \%$; NS) (fig 1). Survival rates remained similar when patients with previous variceal bleeding or with associated fundal varices were excluded from the analysis (data not shown).

Multivariate analysis showed that among the variable tested (treatment group, age, sex, alcoholism, Pugh score, and treatment group), Pugh score was the only independent predictor of survival (odds ratio 1.6; 95\% CI 1.1-2.2; $\mathrm{p}<0.005)$.

REBLEEDING

The causes of rebleeding are indicated in table 3. Rebleeding of non-variceal sources was observed in six patients in the TIPS group and in nine in the ligation group.

Nine episodes of variceal rebleeding were observed in eight patients in the TIPS group $(19.5 \%)$, as a result of stent stenosis in six patients and late shunt thrombosis in two patients. Stent stenosis was treated by shunt dilatation and shunt thrombosis by reopening of the shunt and insertion of an additional stent.

Thirty episodes of variceal bleeding occurred in 22 patients in the ligation group $(56.4 \%)$. Early rebleeding (within 10 days) was observed in 11 patients. Rebleeding was uncontrolled in 11 patients (oesophageal varices, eight patients; gastric varices, three patients); eight patients were treated by emergency TIPS while three patients died from massive haemorrhage associated with end stage liver failure. The variceal rebleeding rate at two years was significantly lower in patients in the

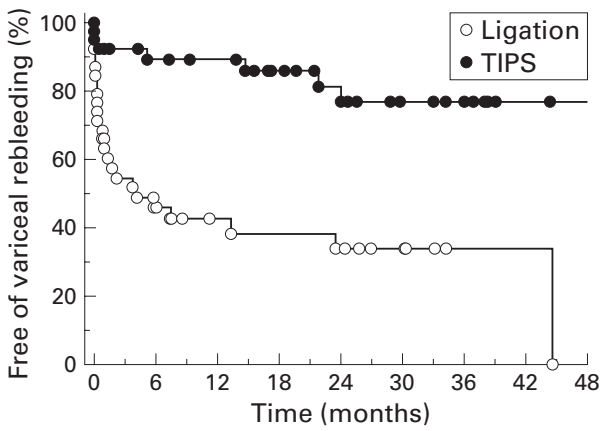

No of patients at risk

$\begin{array}{llllllllll}\text { Banding } & 39 & 15 & 10 & 9 & 8 & 5 & 1 & 1 & 0\end{array}$ $\begin{array}{llllllllll}\text { TIPS } & 41 & 31 & 29 & 23 & 19 & 11 & 11 & 9 & 1\end{array}$

Figure 2 Cumulative proportion of patients free of variceal bleeding (Kaplan-Meier plot) in the ligation and transjugular intrahepatic portosystemic shunt (TIPS) groups.

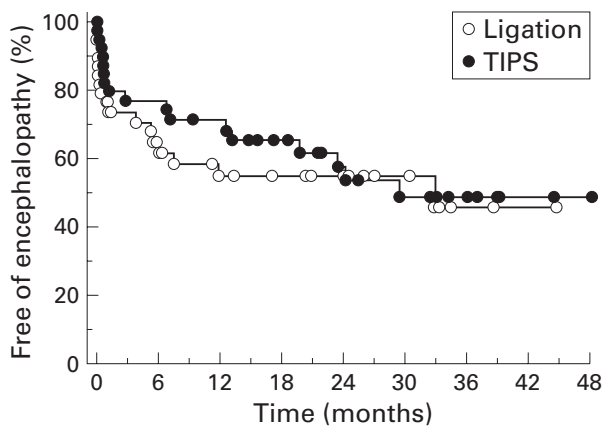

No of patients at risk

$\begin{array}{llllllllll}\text { Banding } & 39 & 21 & 16 & 13 & 11 & 7 & 3 & 2 & 1\end{array}$ $\begin{array}{llllllllll}\text { TIPS } & 41 & 24 & 19 & 14 & 10 & 7 & 5 & 2 & 2\end{array}$

Figure 3 Cumulative proportion of patients free of hepatic encephalopathy (Kaplan-Meier plot) in the ligation and transjugular intrahepatic portosystemic shunt (TIPS) groups.

TIPS group compared with the ligation group (18.5\% v 66\%; $\mathrm{p}<0.001$ ) (fig 2).

Multivariate analysis showed that among the variables tested (treatment group, age, alcoholism, severity of index bleed as reflected by the number of blood units transfused, time interval between index bleed, and randomisation), treatment by TIPS was the only significant predictor of the rate of variceal rebleeding (odds ratio $0.08 ; 95 \%$ CI $0.02-0.2 ; \mathrm{p}<0.0001$ ).

ENCEPHALOPATHY

Encephalopathy was present on the day of randomisation in seven patients in the TIPS group and in seven in the ligation group.

During follow up, encephalopathy was observed in 16 patients (mild, 13 patients; moderate, one patient; severe, two patients) in the ligation group and in 15 (mild, seven patients; moderate, six patients; severe, two patients) in the TIPS group. The cumulative probability of developing encephalopathy during follow up was not significantly different between the two groups at two years: $44 \%$ for the ligation group compared with $47 \%$ for the TIPS group (fig 3). Multivariate analysis showed that none of the variables tested (treatment group, age, sex, alcoholism, Pugh score, pre-TIPS hepatic encephalopathy) was an 
Table 4 Randomised clinical trials comparing transjugular intrahepatic portosystemic shunt (TIPS) and endoscopic and/or pharmacological therapy

\begin{tabular}{|c|c|c|c|c|c|c|c|c|c|c|c|c|}
\hline \multirow[b]{2}{*}{ Study (ref) } & \multicolumn{2}{|c|}{ Patients (n) } & \multicolumn{2}{|c|}{$\begin{array}{l}\text { Time to } \\
\text { randomisation } \\
\text { (days) }\end{array}$} & \multicolumn{2}{|c|}{ Pugh class $(A / B / C)(n)$} & \multicolumn{2}{|c|}{$\begin{array}{l}\text { Variceal rebleeding } \\
(1 \text { year })(\%)\end{array}$} & \multicolumn{2}{|c|}{$\begin{array}{l}\text { Encephalopathy } \\
\text { (1 year) (\%) }\end{array}$} & \multicolumn{2}{|c|}{$\begin{array}{l}\text { Death (1 year) } \\
(\%)\end{array}$} \\
\hline & Controls & TIPS & Controls & TIPS & Controls & TIPS & Controls & TIPS & Controls & TIPS & Controls & TIPS \\
\hline${ }^{\mathrm{a}}$ Cello et $a l^{21}$ & 25 & 24 & 1.5 & 1.5 & - & - & $48^{\star \mathrm{e}}$ & $13^{\mathrm{e}}$ & $44^{\mathrm{e}}$ & 50 & 32 & 33 \\
\hline${ }^{\mathrm{c}}$ Sauer et $a l^{66}$ & 41 & 42 & 1.2 & 1.1 & $12 / 18 / 11$ & $15 / 18 / 9$ & $44^{\star}$ & 9 & $13^{\star}$ & 26 & 15 & 23 \\
\hline${ }^{\mathrm{a}}$ Cabrera et a ${ }^{20}$ & 32 & 31 & 3.0 & 3.0 & $14 / 16 / 2$ & $14 / 13 / 4$ & $52^{\star}$ & 23 & $13^{\star}$ & 33 & 18 & 7 \\
\hline${ }^{\circ}$ GEAIH et al ${ }^{9}$ & 33 & 32 & $<4$ & $<4$ & $0 / 0 / 33$ & $0 / 0 / 32$ & $61^{\star}$ & 41 & - & - & 42 & 50 \\
\hline${ }^{\mathrm{b}}$ Sauer et $^{2 l^{8}}$ & 17 & 16 & - & - & - & - & $54^{\star}$ & 18 & 12 & 25 & 9 & 11 \\
\hline${ }^{\mathrm{d}}$ Escorcell et al ${ }^{\beta^{0}}$ & 44 & 47 & 5.0 & 5.0 & $0 / 44(B+C)$ & $0 / 47(B+C)$ & $27^{\star}$ & 11 & $4^{\star}$ & 32 & 25 & 28 \\
\hline balan et al ${ }^{2}$ & 27 & 31 & 1.0 & 1.0 & $5 / 9 / 13$ & $2 / 14 / 15$ & $52^{\star}$ & 9.8 & 26 & 35 & 30 & 31 \\
\hline $\begin{array}{l}{ }^{\mathrm{a}} \text { Garcia-Villareal } \\
\text { et al }\end{array}$ & 24 & 22 & 5.6 & 5.4 & $3 / 14 / 7$ & $5 / 10 / 7$ & $50^{\star}$ & 9 & 25 & 23 & $18^{\star}$ & 0 \\
\hline${ }^{\mathrm{a}}$ Merli et $a l^{4}$ & 43 & 38 & $1-180$ & $\begin{array}{l}1-18 \\
0\end{array}$ & $13 / 25 / 5$ & $13 / 20 / 5$ & $52^{\star}$ & 21 & $18^{\star}$ & 50 & 14 & 16 \\
\hline${ }^{\mathrm{b}}$ Rossle et $a l^{25}$ & 65 & 61 & 4.4 & 6.3 & $22 / 31 / 12$ & $17 / 33 / 11$ & $41^{\star}$ & 15 & $18^{\star}$ & 36 & 10 & 11 \\
\hline${ }^{a}$ Sanyal et $a l^{27}$ & 39 & 41 & - & - & $6 / 15 / 18$ & $7 / 13 / 21$ & 21 & 23 & $13^{\star}$ & 29 & 10 & 28 \\
\hline${ }^{b}$ Present study & 39 & 41 & 1.7 & 1.8 & $0 / 17 / 22$ & $0 / 20 / 21$ & $57^{\star}$ & 11 & 40 & 32 & 38 & 25 \\
\hline
\end{tabular}

Control group: asclerotherapy; band ligation; ${ }^{\mathrm{c}}$ sclerotherapy and propranolol; isosorbide mononitrate and propranolol; ${ }^{\mathrm{e}}$ prevalence during follow up.

GEAIH, Groupe d'Étude des Anastomoses Intra-Hépatiques.

${ }^{\star} \mathrm{p}<0.05$ compared with TIPS.

independent predictor of occurrence of encephalopathy after randomisation.

SHUNT DYSFUNCTION

During follow up, 30 episodes of shunt dysfunction were observed in 24 patients. The cumulative probability of developing shunt dysfunction was $73 \%$ at two years.

DURATION OF HOSPITAL STAY

Duration of hospitalisation for the index bleed was 13.4 (2.4) days in the ligation group compared with 15.8 (1.8) days (mean (SD)) in the TIPS group (NS). During a two year follow up, the number of hospitalisation days per patient was similar in the ligation and TIPS groups (40 (6) days $v 45$ (7) days; mean (SD)).

\section{Discussion}

Variceal bleeding is associated with a high rate of rebleeding and death within months after the index bleed. In recent years, the use of endoscopic sclerotherapy or ligation, and $\beta$ blockers has markedly lowered the rebleeding rate but the effect on survival has been more modest. ${ }^{17}$ The TIPS procedure was first used in 1989 and initial results were encouraging. ${ }^{18} 19$

Treatment of variceal bleeding is an emergency due to the frequency and severity of early rebleeding during the first two weeks. Early intervention is the only way to significantly influence survival. Therefore, patients were randomised 24 hours after haemodynamic stabilisation, achieved in both groups by standard treatment for acute variceal bleeding (that is, vasoactive drugs and endoscopic therapy). The TIPS procedure was performed soon after randomisation whereas the second endoscopic treatment was delayed $7-10$ days to avoid occurrence of bleeding from ligation related ulcers.

Additional considerations which led to the present study design need to be discussed. We introduced the TIPS procedure in our centre in 1991 but we did not initiate the present study until 1994 to avoid the possible influence of a learning effect on the results. Patients with good liver function were excluded from the study as TIPS was considered experimental and was not proposed for patients with a good life expectancy, such as Pugh class A, when the study was initiated. Patients with end stage liver failure (Pugh score $>12$ ) were also excluded because it is well demonstrated that survival of these patients is poor irrespective of the treatment used to control variceal bleeding. The study was conducted as a single centre trial to avoid bias that may result from differences in technical expertise in different centres and also to control for the accuracy of critical information such as rebleeding, occurrence of encephalopathy, and cause of death. Variceal band ligation was used instead of endoscopic sclerotherapy as ligation is superior to sclerotherapy in terms of number of sessions needed for obliteration, need for blood transfusion, and incidence of symptomatic related ulcers. ${ }^{11}$ Survival was chosen as the main end point. Values used to calculate sample size were derived from the only large series of variceal bleeding treated by TIPS available in $1994^{18}$ where the two year survival rate in Pugh class B and C patients was reported to be $80 \%$. A $50 \%$ survival rate in the banding group was assumed according to our former experience with endoscopic sclerotherapy in a large group of Pugh B and C cirrhotic patients (unpublished results).

Eleven comparative studies evaluating the effect of TIPS in the treatment of variceal bleeding have been reported; eight have been published as full papers and three in abstract form $^{20-30}$ These studies had different designs and involved different patient populations. TIPS was compared with endoscopic sclerotherapy alone (five studies), with sclerotherapy in combination with propranolol (three studies), with variceal ligation (two studies), and with propranolol and isosorbide mononitrate (one study). The timing of randomisation and treatment were highly variable as were the definitions of rebleeding, encephalopathy, treatment failure, and TIPS dysfunction.

Therefore, survival and rebleeding rates as well as the incidence of hepatic encephalopathy after randomisation were highly variable among the different studies. Despite this heterogeneity, comparisons between TIPS and endoscopic therapy gave similar results in 
terms of survival and variceal rebleeding but controversy exists with regard to the incidence of encephalopathy (table 4).

In the present study, the survival rate was not significantly different in the TIPS group compared with the ligation group. In the literature, one year survival rates in the TIPS and control groups varied widely $(0-42 \%)$; however, it was similar for both groups in nine studies, ${ }^{20-22}{ }^{24-26}{ }^{28-30}$ worse in the TIPS group in one,$^{27}$ and one study reported improved survival with TIPS. ${ }^{23}$ Not surprisingly, Pugh score at randomisation was the major determinant of survival.

We observed a high frequency of rebleeding in the banding group. This may be explained by the characteristics of our population (Child B and C) and by our definition of rebleeding (any rebleeding episode occurring 24 hours after randomisation was considered rebleeding). The rate of variceal rebleeding observed at one year $(57 \%)$ was similar to that reported by others who used the same criteria as ours. ${ }^{21-23} 2629$ The variceal rebleeding rate was much lower after TIPS than after variceal ligation. This finding was confirmed by all but one study published previously. ${ }^{27}$ Multivariate analysis demonstrated that treatment with TIPS was the only parameter associated with decreased variceal rebleeding rate.

There are discrepancies with regard to the incidence of hepatic encephalopathy following treatment which was found to be increased in the TIPS group in six studies..$^{24-27}{ }^{30}$ The true rate of encephalopathy is difficult to evaluate among previously published studies. In some papers, only spontaneous encephalopathy was considered; in others new or aggravated encephalopathy was only recorded. In the present study, encephalopathy occurred frequently in both the ligation and TIPS groups probably because all patients had moderate or severe liver failure, as observed in other studies where the rate of encephalopathy was similar for both treatment groups. ${ }^{21-23} 2829$ Multivariate analysis demonstrated that none of the variables tested (including treatment group) allowed prediction of encephalopathy during follow up.

Treatment failure occurred in $11 / 39$ patients $(28 \%)$ treated by ligation, a value similar to the rate observed in previous studies. ${ }^{20-29}$ The majority of patients with treatment failure were crossed over to TIPS with the exception of the study of Garcia-Villareal and colleagues ${ }^{23}$ which may explain, at least in part, the better survival rate observed in the TIPS group.

The incidence of shunt dysfunction is more difficult to assess given the differences in definitions of dysfunction (angiographic versus haemodynamic) and the schedule of angiography with pressure measurements during follow up. However, it is clear that dysfunction occurs frequently during the first year (probably in $60-80 \%$ of patients), as demonstrated by the present trial, and that recurrence of stenosis after a first episode is also frequent. Surprisingly, the rebleeding rate remains low despite the high incidence of shunt dysfunction suggesting that in many patients the manomet- ric threshold for rebleeding may be higher than the classical $12 \mathrm{~mm} \mathrm{Hg}$ pressure level.

From analysis of 10 comparative trials of TIPS versus endoscopic therapy it can be concluded that both treatments are equivalent in terms of survival and that the beneficial effect of TIPS on rebleeding might be counterbalanced by an increased incidence of encephalopathy. ${ }^{31}$ The number of reinterventions for shunt insufficiency must also be balanced against the number of endoscopies required to eradicate varices and to monitor variceal recurrence. It should also be mentioned that in the majority of studies, sclerotherapy failures were treated by TIPS which may have obscured the beneficial effect of TIPS on survival given the use of intent to treat analysis.

If variceal ligation and TIPS are to be first line therapies for variceal bleeding in cirrhotic patients with moderate or severe liver failure, future studies must evaluate important issues such as cost effectiveness and quality of life as primary end points. Costs were reported to be similar for TIPS and sclerotherapy in two studies $^{2021}$ whereas Meddi and colleagues ${ }^{32}$ demonstrated that prevention of variceal rebleeding with TIPS was not a cost saving strategy in comparison with sclerotherapy. A recent study suggested that TIPS improves the quality of life but comparison with a group of patients treated with sclerotherapy or ligation was lacking. ${ }^{33}$ Finally, the choice of therapy to prevent rebleeding must be done according to the local facilities and expertise available in each centre.

The present study was supported in part by a grant from the Medical Research Council of Canada (UI 11508).

1 Smith JL, Graham DY. Variceal hemorrhage. A critical evaluation of survival analysis, Gastroenterology 1982;82:962-73

2 Graham DY, Smith JL. The course of patients after variceal hemorrhage. Gastroenterology 1981;80:800-9.

3 Pugh RNH, Murray-Lyon IM, Dawson JL, et al. Transection of the oesophagus for bleeding oesophageal varices. $B r$ tion of the oesophagus
S $1973 ; 60: 646-9$

4 Infante-Rivard C, Esnaola S, Villeneuve JP. Clinical and statistical validity of conventional prognostic factors in predicting short-term survival among cirrhotics. Hepatology
pacticas 987;7:660-4

5 Orloff MJ, Bell RH. Long-term survival after emergency portacaval shunting for bleeding varices in patients with alcoholic cirrhosis. Am f Surg 1986;151:176-83

6 Cello JP, Grendell JH, Grass RA, et al. Endoscopic sclerotherapy versus portacaval shunt in patients with severe cirrhosis and variceal hemorrhage. $N$ Engl $7 \mathrm{Med}$ 1984;311:1589-94.

7 Villeneuve JP, Infante-Rivard C. Prevention of variceal rebleeding by propranolol: should it work? Does it work? Hepatology 1997;7:1386-8

8 Villanueva C, Balanzo J, Novella MT, et al. Nadolol plus isosorbide mononitrate compared with sclerotherapy for the prevention of variceal rebleeding. $N$ Engl $\mathscr{f}$ Med 1996;334:1624-9.

9 Bernard B, Lebrec D, Mathurin P, et al. Beta-adrenergic antagonists in the prevention of gastrointestinal rebleeding in patients with cirrhosis: a meta-analysis. Hepatology 1997; in patients

10 Infante-Rivard C, Esnaola S, Villeneuve JP. Role of endoscopic variceal sclerotherapy in the long-term management of variceal bleeding. A meta-analysis. Gastroenterology 1989;96:1087-92.

11 Laine L, Cook D. Endoscopic ligation compared with sclerotherapy for treatment of oesophageal variceal bleeding. A meta-analysis. Ann Intern Med 1995;123:280-7.

12 Richter GM, Noeldge G, Palmaz JC, et al. Transjugular intrahepatic portacaval stent shunt: preliminary clinical results. Radiology 1990;174:1027-30.

13 Peto R, Pike MC, Armitage P, et al. Design and analysis of randomized clinical trials requiring prolonged observations. II. Analysis and examples. Br f Cancer 1977;35:1-39. 4 Bilodeau $M$, Rioux L, Willems $B$, et al. Transjugular intrahepatic portacaval stent shunt as a rescue treatment with severe liver failure. Am f Gastroenterol 1992;87:36971 . 
15 Lafortune M, Martinet JP, Denys A, et al. Short and long-term hemodynamic effects of TIPS: a Doppler/

16 Fleiss JL. Statistical methods for rate and proportion, 2nd edn New York: Wiley Interscience, 1981

17 D'Amico G, Pagliaro L, Bosch J. The treatment of portal hypertension. A meta-analytic review. Hepatology 1995;22 332-54.

18 Rossle M, Haag K, Ochs A, et al. The transjugular intrahepatic portosystemic stent shunt procedure for variceal bleeding. $N$ Engl f Med 1994;330:165-71.

19 Laberge JM, Ring ES, Gordon RL, et al. Creation of transjugular intrahepatic portosystemic shunts with the Wallstent endosprosthesis: results in 100 patients, Radiology 1993;185:813-17.

20 Cabrera J, Maynar M, Granados R, et al. Transjugular intrahepatic portosystemic shunt versus sclerotherapy in the elective treatment of variceal hemorrhage. Gastroenterology 1996;110:832-9.

21 Cello JP, Ring EJ, Olgott EW, et al. Endoscopic sclerotherapy compared with percutaneous transjugular intrahepatic portosystemic shunt after initial sclerotherapy in patients with acute variceal hemorrhage. A randomized,

22 Jalan R, Forrest EH, Stanley AJ, et al. A randomized trial comparing transjugular intrahepatic portosystemic stentshunt with variceal band ligation in the prevention of rebleeding from esophageal varices. Hepatology 1997;26: 1115-22.

23 Garcia-Villareal L, Martinez-Lagares F, Sierra A, et al. Transjugular intrahepatic portosystemic shunt versus endoscopic sclerotherapy for the prevention of variceal rebleeding after recent variceal hemorrhage. Hepatology 1999;29:27-32.

24 Merli M, Salerno F, Riggio O, et al. Transjugular intrahepatic portosystemic shunt versus endoscopic sclerotherapy for the prevention of variceal bleeding in cirlerotherapy for the prevention of variceal bleeding in cirrhosis:

25 Rossle M, Deibert P, Haag K, et al. Randomised trial of Rossle M, Deibert P, Haag K, et al. Randomised trial of
transjugular intrahepatic portosystemic shunt versus en- doscopy plus propranolol for prevention of variceal rebleeding. Lancet 1997;349:1053-9.

26 Sauer P, Theilman L, Stremmel W, et al. Transjugular intrahepatic portosystemic shunt versus sclerotherapy plus propranolol for variceal rebleeding. Gastroenterology 1997;113: 1623-31.

27 Sanyal AJ, Freedman AM, Luketic VA, et al. Transjugular intrahepatic portosystemic shunts compared with endoscopic sclerotherapy for the prevention of recurrent variceal hemorrhage. A randomized controlled trial. Ann Intern Med 1997;126:849-57.

28 Sauer P, Theilman L, Benz C, et al. Transjugular intrahepatic portosystemic stent shunt (TIPS) vs endoscopic banding in the prevention of variceal rebleeding: interim analysis of a randomized study. Gastroenterology 1997;112:A1374.

29 Groupe d'Étude des Anastomoses intra-hépatiques: TIPS vs sclerotherapy: preliminary results of a multicenter randomized trial. Hepatology 1995;22 (part 2): 297A.

30 Escorsell A, Banares R, Gilabert R, et al. Transjugular intrahepatic portosystemic shunt (TIPS) versus propranolol + isosorbide - mononitrate $(\mathrm{P}+\mathrm{I})$ for the prevention of variceal rebleeding in patients with cirrhosis. Results of a randomized controlled trial. Hepatology 1998;8(suppl): $770 \mathrm{~A}$.

31 Papatheodoridis GV, Goulis J, Leandro G, et al. Transjugular intrahepatic portosystemic shunt compared with endoscopic treatment for prevention of variceal rebleeding: a metaanalysis. Hepatology 1999;30:612-22.

32 Meddi P, Merli M, Lionetti R, et al. Cost analysis for the prevention of variceal rebleeding: a comparison between transjugular intrahepatic portosystemic shunt and endoscopic sclerotherapy in a selected group of Italian cirrhotic patients. Hepatology 1999;29:1074-7.

33 Nazarian GK, Ferral H, Bjarwason H, et al. Effect of transjugular intra-hepatic portosystemic shunt on quality of life. Am F Roentgenol 1996;167:963-9. 